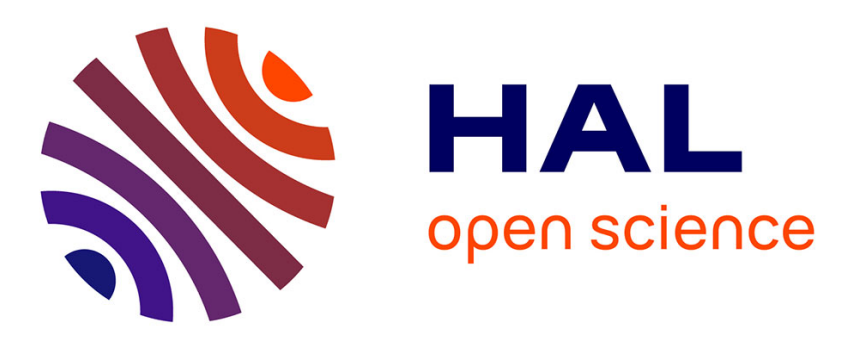

\title{
Microfluidic directional emission control of an azimuthally polarized radial fibre laser
}

\author{
Alexander M. Stolyarov, Lei Wei, Ofer Sharipa, Fabien Sorin, Song L. Chua, \\ John D. Joannopoulos, Yoel Fink
}

\section{- To cite this version:}

Alexander M. Stolyarov, Lei Wei, Ofer Sharipa, Fabien Sorin, Song L. Chua, et al.. Microfluidic directional emission control of an azimuthally polarized radial fibre laser. Nature Photonics, 2012, 6, pp.229-233. hal-00666119

\section{HAL Id: hal-00666119 https://hal.science/hal-00666119}

Submitted on 3 Feb 2012

HAL is a multi-disciplinary open access archive for the deposit and dissemination of scientific research documents, whether they are published or not. The documents may come from teaching and research institutions in France or abroad, or from public or private research centers.
L'archive ouverte pluridisciplinaire HAL, est destinée au dépôt et à la diffusion de documents scientifiques de niveau recherche, publiés ou non, émanant des établissements d'enseignement et de recherche français ou étrangers, des laboratoires publics ou privés. 


\section{$\underline{\text { Microfluidic directional emission control of an azimuthally polarized }}$}

\section{radial fibre laser}

Alexander M. Stolyarov ${ }^{1,2,3}$, Lei Wei ${ }^{1,2}$, Ofer Shapira ${ }^{1,2}$, Fabien Sorin ${ }^{1,2,4}$, Song L.

Chua $^{1,5}$, John D. Joannopoulos ${ }^{1,2,6}$, and Yoel Fink ${ }^{1,2,7^{*}}$

${ }^{1}$ Research Laboratory of Electronics, Massachusetts Institute of Technology, 77

Massachusetts Avenue, Cambridge, Massachusetts 02139, USA

${ }^{2}$ Institute for Soldier Nanotechnologies, Massachusetts Institute of Technology, 77

Massachusetts Avenue, Cambridge, Massachusetts 02139, USA

${ }^{3}$ School of Engineering and Applied Sciences, Harvard University, 29 Oxford Street,

Cambridge, Massachusetts 02138, USA

${ }^{4}$ Current address: Laboratoire surface du verreet interfaces, UnitéMixte CNRS/Saint-

Gobain UMR 125, 39 quai Lucien Lefranc, 93303 Aubervilliers, France

${ }^{5}$ Department of Electrical Engineering and Computer Science, Massachusetts Institute of

Technology, 77 Massachusetts Avenue, Cambridge, Massachusetts 02139, USA

${ }^{6}$ Department of Physics, Massachusetts Institute of Technology, 77 Massachusetts

Avenue, Cambridge, Massachusetts 02139, USA

${ }^{7}$ Department of Materials Science and Engineering, Massachusetts Institute of

Technology, 77 Massachusetts Avenue, Cambridge, Massachusetts 02139, USA

*corresponding author; email: yoel@mit.edu 
Lasers with cylindrically symmetric polarization statesare predominantly basedon whispering-gallerymodes ${ }^{1-7}$, characterized by high angular momentum and dominated by azimuthal emission.Here azero angular momentum laserwith purely radial emission is demonstrated.An axially invariant, cylindrical photonic bandgap fibrecavity ${ }^{8}$ filled with a microfluidic gain medium plug is axially pumped, resulting in a unique radiating field pattern characterized by cylindrical symmetry and a fixed polarization pointed in theazimuthal direction.Encircling the fibre core is anarray ofelectrically contacted and independently addressable liquid crystal microchannelsembedded in the fibrecladding. Thesechannels modulate the polarized wavefront emanating from the fibre coreleading toa laser with a dynamically controlled intensity distribution spanning the full azimuthal angular range. This new capability,implemented monolithicallywithin a single fibre, presents opportunities ranging from flexible multidirectional displaysto minimally invasive directed light delivery systems for medical applications.

Rotationally symmetric resonators capable of omnidirectional emission in the direction perpendicular to the axis of symmetryhave been the subject of recent studies ${ }^{1-7}$. These cylindrically symmetric sources rely on the excitation of whispering-gallery modes(Fig. 1, bottom), confined near the cavity boundary by total internal reflection. Lightescapes these resonators only through diffraction losses or scattering from surface roughness,thus limiting control over the output coupling. Here we report on a cylindrical photonic bandgap (PBG)fibrecavitywhich supports purely radial modes (Fig. 1, top).In principle, this structure allows full control over output coupling and the potential for reduction in volume without compromising the quality factor. Additionally, the shorter 
cavity length of these radial modes could allow for a larger free spectral range and consequently higher finesse as compared to whispering-gallery modes.Importantly, the radially directed emission from the extended cylindrical surface of this fibre laser is in contrast to the axial emission characteristic of conventional Bragg fibre lasers ${ }^{9,10}$ and planar annular Bragg resonators ${ }^{11-14}$

The fibre fabrication method is based on the thermal drawing of a macroscopic preform (Fig. 2a) assembled with all the solid materials and geometryof the resulting fibre (see Methods section for details). ${ }^{15}$ The preform consists of a hollow annular multilayered cavity comprised of a 35-layerstructure of chalcogenide glass $\left(\mathrm{As}_{25} \mathrm{~S}_{75}\right)$ and polycarbonate (PC). ${ }^{16}$ Further out in the PC cladding are several pairs of conductive carbon-loaded polyethylene (CPE) electrodes which flank hollow channels. The composite structure is drawn under a high stress regime $\left(\sim 400 \mathrm{~g} / \mathrm{mm}^{2}\right)$ yielding an axially invariant, rotationally symmetric, photonic bandgapfibrecavitywith a transmission bandgap centered near550 nm and a bilayer periodicity of $160 \mathrm{~nm}$ (Fig. 2b, top-right). The fibrecore is surrounded by four electrically addressablemicrofluidic channels embedded in the fibre cladding (Fig. 2b, bottom). The challenge in drawing such a structure which is reduced in size by a factor of 100 lies in maintaining the integrity and symmetry of the multilayers down to the final scale while preventing the deformation of the hollow channels and electrodes.

Recent studies have demonstratedoptofluidicfibre devices with dynamically tunable properties ${ }^{17,18}$. In this work, amicrofluidic pump (shown in Fig. 3a and described in Methods section) is utilized to transport organic dye-doped water plugs inside the fibre core. The internal surface of the fibre core is hydrophopic and notwet by the 
aqueousplug, which remains intactduring rapid movement at velocities of $35 \mathrm{~mm} / \mathrm{s}$ (Fig. 3b). The pressure field is mediated through atransparent and immiscible fluid (e.g., silicone oil),allowingreversible transport of the lasing plug to any position within the fibre (Fig. 3b,c).

The laser wavefrontemitted from the fibre core appears as an axially collimated ring-like beam in the far-field (Fig. 4a).The axial collimation results from the low diffraction facilitated by the smooth interfaces of the water plug, and the continuous translational symmetry of the laser cavity along the fibre axis. The penetration depth of the pump beam, combined with the circular cross section of the core, defines a cylinder from which laser light is emitted. (Note that the actual plug length, which is on the order of a cm, does not influence the laser emission). For the 1mM R590 waterplug used in our experiments, the penetration depth is $\sim 100 \mu \mathrm{m}$. Therefore, the axial divergence angle, which is proportional to the ratio between the wavelength and the penetration depth, is expected to be $\sim 10 \mathrm{mrad}$.

Thecylindrical symmetryof the emission stems from the isotropic fluorescence of the dye and its coupling to the fibrecavity modes. Below threshold, the measured isotropic and unpolarized spontaneous emission populates all the available fibre modes. However, above threshold, a purely azimuthally polarized beam emerges from the PBG fibre cavity (Fig. 4b) which indicates coupling to specific low-threshold fibre modes. These modes are the high- $\mathrm{Q} \mathrm{TE}_{0 \mathrm{n}}$ modes with an electric field polarization identical to what we measure in the radial laser emission.This isotropic emission is in contrast to the anisotropic emission observed fromdye-doped solid plugs. ${ }^{20}$ In a low viscosity solvent such as water, fluorescence randomization occurs dueto the fast molecular reorientationof 
the dye, which happens on a picosecond scale ${ }^{21}$, much faster than the nanosecond scale of the fluorescence lifetime ${ }^{22}$. Thus fluorescence anisotropy is not expected, which is consistent with our experimental observations.

A 1mMRhodamine 590 (R590)-dopedwater plugis introduced into the fibre core and pumped by a linearly polarized Nd:YAG laser at $532 \mathrm{~nm}$ (see Methods section for details). A typical measurement depicting the dependence of output energy on input energy shows a clear threshold at $165 \mathrm{~nJ}$ (Fig. 4c). The spacing between individual lasing peaks reveals an average separation of $1.78 \mathrm{~nm}$ for a fibre with an $80 \mu \mathrm{m}$ core (Fig. 4d). Considering the free spectral range of the longitudinal modes in a Fabry-Perot resonator, $\Delta \lambda \approx \lambda_{0}^{2} / 2 n d$, where $\lambda_{0}$ is the central lasing wavelength, and $n$ is the refractive index of the dye solution ( $n=1.33$ ), a cavity length $d=78 \mu \mathrm{m}$ is calculated, which agrees well with the fibre core diameter andfurther confirms the radial nature of the emission.

So how can we use the intrinsic radial emission andazimuthal polarization state to achieve a useful effect? The problem of directing coherent light is recognized as an important one for a variety of applications. Current solutions could be classified into ones that utilize moving mechanical parts and ones that rely on non-mechanical mechanisms ${ }^{23-}$ ${ }^{28}$. While having advantages such as high angular precision, the non-mechanical solutions are nevertheless constrained to a narrow angular range. Here we demonstrate an approach that facilitates directional intensity control withinafull $2 \pi$ radians.

Thus far the hollow microchannels (Fig. 2b) have been empty. But now, we exploit them by infiltrating them with liquid crystals (LCs) in order to create a novel hybrid fibre device capable of modulating the coherent laser emission as a function of angle. The linearly polarized annular wavefront escaping the PBG cavity simultaneously 
interacts with the multiple LCmicrochannels surrounding it, each of which can be independently controlled. In the absence of an applied electric field, the LC director points along the microchannel axis.Applying a potential difference across an electrode pair flanking an LC-infiltrated microchannel generates an electric field perpendicular to the LC director.Above a threshold voltage, the applied field produces a torque on the LC moleculeswhich leads to their rotation ${ }^{29}$. The optical anisotropy of LC molecules together with the control on their spatial orientation provides a mechanism to continuously modify the linearly polarized state of the laser light passing through each microchannel. Fixing a thin external linear polarizer around the fibre, this mechanism is used to modulate the azimuthal laser intensity distribution. We note that although a whispering-gallery-like emission with a precise polarization state could also in principle be modulated by an annular array of LC channels, the non-radial nature of this emission in the vicinity of the cavity would pose significant practical challenges to enable this oblique transmission to be efficiently modulated.

We demonstrate the principle of directional emission control by contacting two oppositely facing LC microchannels toallow for simultaneous and independent tuning of the light intensity emitted from opposite sides of the same fibre (shown in Fig. 5a and described in Methods section).A R590-doped water plug is positioned in the core of the fibre and axially pumped as described previously. A thin polarizer sheet is wrapped around the fibre with its transmission axis aligned along the fibre axis such that the emitted light is blocked in the absence of an applied electric field or below the threshold voltage. Above the threshold voltage, independent intensity modulation of laser light passing through each LC-filled microchannel is clearly visiblein the images (left and right 
sides of Fig. 5a) and is further substantiated by measuring the laser spectra as a function of driving voltage (Fig. 5b). The measurements reveal a maximum extinction ratio of $\sim 9$ $\mathrm{dB}$.

The precise control over the omnidirectional intensity distribution from the surface of a thin and flexible fibre presents a wide range ofintriguing opportunities. The ability to introduce a directionally emitting laser catheter into a blood vessel could enable spatial and angularly selective irradiation of diseased sections while sparing healthy tissue(see Supplementary Fig. S1) in conjunction with treatments such as photodynamic therapy $^{30}$. A lab-in-a-fibre application would involve utilizing the fibre laser to interrogate fluids in the adjacent microchannels. Finally, theintegration of a modulator and light source in a single fibre represents an important step towards the realization of flexible and directional fabric displays (see Supplementary Fig. S2).

\section{Methods:}

Fibre fabrication. The multilayer film is fabricated by thermal evaporation of a $4 \mu \mathrm{m}$ thick $\mathrm{As}_{25} \mathrm{~S}_{75}$ layer onto each side of a $10 \mu \mathrm{m}$ thick PC (Lexan) film. This film is subsequently rolled onto a Teflon lined mandrel and additional thicker PC films are added to increase the outer diameter to $30 \mathrm{~mm}$, after which this preform is consolidated under vacuum at $\sim 190{ }^{\circ} \mathrm{C}$ until the individual layers fuse together into one solid part. After consolidation, groups of three pockets with prescribed dimensions are machined along the length of the preform. The two outer pockets are filled with CPE strips while the center pocket is left empty. Additional PC films are rolled around the preform to a 40 mm diameter and a second consolidation step ensues. During the subsequent thermal 
drawing process, the preform is scaled down by a factor of $\sim 100$, resulting in the vacant pockets being pulled into hollow microchannels, the CPE strips drawn into continuous electrodes which are contacted post draw, and the multilayer glass / polymer microstructure drawn into a nanostructured optical cavity lining the inner core of the fibre.

Microfluidic controls. Bidirectional pumping of fluid into and out of the fibre is achieved by alternating the electrical actuation of microdispensing valves (Lee Company VHS M/2) connected to pressurized and depressurized silicone oil reservoirs. Depressurization is achieved using a standard Venturi-based vacuum generator.

Laser characterization. The organic laser dye Rhodamine 590 (Exciton) dissolved in DI water at $1 \mathrm{mM}$ concentration is infiltrated into the fibre core with a syringe. The pump beam from the second harmonic $(532 \mathrm{~nm})$ of a linearly polarized Nd:YAG laser (Continuum Minilite II) with a nominal pulse duration of $9 \mathrm{~ns}$ and $10 \mathrm{~Hz}$ repetition rate is coupled into the fibre core through a $10 \mathrm{~cm}$ focal length lens. A small fraction of the pump beam is diverted with a beam splitter to monitor the input pump energy using a laser energy meter (Molectron EPM 1000 with the J4-09 probe). A 10x objective lens is used to collect a fraction of the light emitted radially from the fibre and focused directly onto the entrance slit of a spectrometer (Ocean Optics USB4000-UV-VIS). The polarization of the emitted light is measured by rotating a linear polarizer positioned between the fibre and the spectrometer. 
Intensity modulation using LCs.LCs (Merck MLC-2058) are introduced into themicrochannelsby capillary action, resulting in the LC director aligning parallel to the fibre axis. A function generator (Stanford Research Systems DS345) operated at a sinusoidal $100 \mathrm{~Hz}$ is used to drive a high voltage amplifier (A. A. Lab Systems A-301) which is connected to the electrodes. A 10x objective lens is used to image each LC-filled microchannel to a CCD camera (MoticMoticam 1000) and a series of images are recorded as the voltage is changed. The CCD camera is replaced with a spectrometer to measure the laser spectra.

\section{References:}

[1]Chang, R. K.\&Campillo, A. J.Optical Processes in Microcavities(World Scientific, Singapore, 1996).

[2] McCall, S. L., Levi, A. F. J.,Slusher,R. E.,Pearton, S. J. \&Logan, R.A. Whisperinggallery mode microdisk lasers. Appl. Phys. Lett.60, 289-291 (1992).

[3] Knight, J. C., Driver, H. S. T., Hutcheon, R. J. \& Robertson, G. N. Core-resonance capillary-fiber whispering-gallery-mode laser. Opt. Lett.17, 1280-1282 (1992).

[4] Kawabe, Y. et al. Whispering-gallery-mode microring laser using a conjugated polymer. Appl. Phys. Lett.72, 141-143 (1998).

[5] Moon, H.-J., Chough, Y.-T. \&Kyungwon A. Cylindrical microcavity laser based on the evanescent-wave-coupled gain. Phys. Rev. Lett.85, 3161-3164 (2000). 
[6] Malko, A. V. et al. From amplified spontaneous emission to microringlasing using nanocrystal quantum dot solids. Appl. Phys. Lett.81, 1303-1305 (2002).

[7] Kazes, M., Lewis, D. Y., Ebenstein, Y., Mokari, T. \&Banin, U. Lasing from Semiconductor Quantum Rods in a Cylindrical Microcavity. Adv. Mater.14, 317-321 (2002).

[8] Yeh, P., Yariv, A.\&Marom, E.Theory of Bragg fiber.J. Opt. Soc. Am. 68, 1196-1201 (1978).

[9] Ball, G. A. \& Morey, W. W. Continuously tunable single-mode erbium fiber laser. Opt. Lett.17, 420-422 (1992).

[10] Dianov, E.M., Likhachev, M.E., Fevrier, S. Solid-core photonic bandgap fibers for high-power fiber lasers. IEEE J. Sel. Top. Quant. Electron. 15, 20-29 (2009).

[11] $\mathrm{Wu}, \mathrm{C}$. et al. Optically pumped surface-emitting DFB GaInAsP/InP lasers with circular grating. Electron.Lett.27, 1819-1820 (1991).

[12] Erdogan, T. et al. Circularly symmetric operation of a concentric-circle-grating, surface-emitting, AlGaAs/GaAs quantum-well semiconductor laser. Appl. Phys. Lett.60, 1921-1923 (1992).

[13] Labilloy, D. et al. High-finesse disk microcavity based on a circular Bragg reflector. Appl. Phys. Lett.73, 1314-1316 (1998).

[14] Scheuer, J., Green, W. M. J., DeRose, G. \&Yariv, A., Low-threshold twodimensional annular Bragg lasers. Opt. Lett.29, 2641-2643 (2004).

[15] Abouraddy, A. F. et al. Towards multimaterial multifunctional fibres that see, hear, sense and communicate. Nature Mater.6, 336-347 (2007). 
[16] Ruff, Z. et al. Polymer-composite fibers for transmitting high peak power pulses at 1.55 microns. Opt. Express 18, 15697-15703 (2010).

[17] Alkeskjold, T. T. et al. Integrating liquid crystal based optical devices in photonic crystal fibers. Opt. Quant. Electron.39, 1009-1019 (2007).

[18] Kuhlmey, B. T., Eggleton, B. J. \& Wu, D. K. C. Fluid-Filled Solid-Core Photonic Bandgap Fibers. J. Lightwave Technol.27, 1617-1630 (2009).

[19] Rowland, K. J., Afshar V., A., Monro, T. M., Stolyarov, A. \& Fink, Y. Bragg Waveguides with Low-Index Liquid Cores.Opt. Express20, 48-62 (2012).

[20] Shapira, O. et al. Surface-emitting fiber lasers. Opt. Express14, 3929-3935 (2006). [21] Eichler, H. J., Klein, U. \&Langhans, D. Measurement of orientational relaxation times of Rhodamine 6G with a streak camera. Chem. Phys. Lett.67, 21-23 (1979). [22] Duarte, F. J. \& Hillman, L. W. Dye Laser Principles.(Academic Press Inc., Boston, MA, 1990).

[23] Scifres, D. R., Streifer, W. \& Burnham, R. D. Beam scanning with twin-stripe injection lasers. Appl. Phys. Lett.33, 702-704 (1978).

[24] P. F. McManamon, P. F. et al.Optical Phased Array Technology.Proc. IEEE 84, 268298 (1996).

[25] Wang, X, Wilson, D., Muller, R., Maker, P. \&Psaltis, D."Liquid-crystalblazedgrating beam deflector. Appl. Opt.39,6545-6555 (2000).

[26] Choi, M., Tanaka, T., Fukushima, T. \&Harayama, T. Control of directional emission in quasistadiummicrocavity laser diodes with two electrodes. Appl. Phys. Lett.88, 211110 (2006). 
[27] Smith,N. R., Abeysinghe, D. C., Haus, J. W. \&Heikenfeld, J. Agile wide-angle beam steering withelectrowettingmicroprisms. Opt. Express14, 6557-6563 (2006).

[28] Kurosaka, Y. et al. On-chip beam-steering photonic-crystal lasers. Nature Photon.4, 447-450 (2010).

[29]Yeh, P. \&Gu, C. Optics of Liquid Crystal Displays, second ed. (John Wiley \& Sons, Inc., Hoboken, New Jersey, 2010).

[30] Dolmans, D. E., Fukumura, D. \& Jain, R. K. Photodynamic therapy for cancer.Nature Rev. Cancer3, 380-387 (2003).

Figure 1 |Comparison between radial and whispering-gallery modes.(top) Schematic drawing of the radially emitting fibre laser structure and energy density plot for a high-Q $\mathrm{TE}_{\text {0n }}$ fibre cavity laser mode. The outgoing,radially-directed red arrows denote the direction of laser emission. The white arrows overlaid on the energy density plot denote the polarization of the mode, which is also indicated in black arrows outside the cavity on the schematic. The periodic PBG structure surrounding the fibre core corresponds to layers of $\mathrm{As}_{25} \mathrm{~S}_{75}$ (purple) and polycarbonate (yellow). (bottom) Schematic drawing of a cylindrical structure which supports high-Q whispering-gallery modes. The primarily azimuthally-directed red arrows denote the tangential laser emission of the high angular momentum modes. The energy density plot corresponds to a high order whisperinggallery mode supported by the cylindrical structure shown in blue. The polarization of this mode is indicated by the white dots overlaid on the energy density plot and the black dots on the schematic. Note: the color scheme indicated in the figure applies to both top and bottom energy density plots. 
Figure 2 |Preform and fibre structure.a, Photograph of the drawn preform. The black strips are the CPE electrodes. The orange reflection from $\mathrm{As}_{25} \mathrm{~S}_{75}$ glass is seen in the center of the structure. (inset) Several meters of fibreis coiled around a US quarter. b, (bottom) Scanning electronmicroscope (SEM) micrograph of the multimaterialfibre device. The fibre core is surrounded by four light modulators. (top left) Zoom view of the CPE electrodes flanking a hollow microchannel, which is filled with LCs post draw. (top right) Zoom view of the multilayer structure forming the PBG cavity. The white and grey layers correspond to $\mathrm{As}_{25} \mathrm{~S}_{75}$ and $\mathrm{PC}$, respectively.

Figure 3 | Microfluidic laser system.a, Schematic of the microfluidic system used to transport the gain medium plug within the fibre core.The system is composed of an optical / fluidic coupler at the input facet of the fibre and a set of pressure driven, electrically actuated microdispensing valves at the distal end, allowing for bidirectional flow control.Silicone oil (refractive index, $\mathrm{n}=1.38$ )is in light blue and the organic dyedoped water plug (refractive index, $\mathrm{n}=1.33$ ) is the red cylinder.b, The dynamics of the system is recorded as the plug is optically pumped and simultaneously set into an oscillating motion by alternating the valve actuations. The blue circles correspond to the measured plug position. The black curve is a sinusoidal fit to the data. Plug velocities up to $35 \mathrm{~mm} / \mathrm{s}$ are achieved before the drop disperses into two or more fragments.c, Photographs of the lasing plug at two different positions along the fibre. The radially emitted laser light is seen scattering from a screen with a "rle" logo positioned behind the 
fibre. (The green pump light is also seen along the fibre axis. This is due to the transmission bandgap shift, which arises from filling the core with the silicone oil. ${ }^{19}$ )

Figure 4 | Laser characterization.a, (top) Schematic of the fibre laser threaded through a funnel, the surface of which is used to register the radially emitted wavefront. The red cylinder in the fibre core is a Rhodmaine 590 (R590)-doped water plug. (bottom) Picture of the fibre threaded through the funnel. The radially emitted light emanating from the dye-doped plugis seen as a ring-like pattern scattered from the inner surface of the funnel. The green light at the center is from the pump beam.b,Polarizationof the radial laser light emitted from the fibre, measured by rotating a linear polarizer in the plane shown by the vectors in the inset. c, (left) Output energy dependence on pump energy for a R590-doped water plug showing a threshold of $165 \mathrm{~nJ}$. (right) Spectra measured from the fibre laser below threshold (A), near threshold (B), and above threshold (C). d,Resolved spectra from the emission of an $80 \mu \mathrm{m}$ core fibre just above the laser threshold.The measured quality factor, given by $Q=\lambda_{0} / \delta \lambda$ (where $\delta \lambda$ is the spectral width of a given mode) is 615 ; this is lower than expected from theory, which is due to the limited resolution of the measurement setup and a non-ideal fibre structure.

Figure 5 | Azimuthally controlled laser emission.a, Schematic of the fibre device depicting the simultaneous control of two oppositely facing LC-filled microchannels, labeled as $\mathrm{LC}_{1}$ and $\mathrm{LC}_{2}$. The light blue strips correspond to the $\mathrm{LC}$-filled microchannels and the black strips correspond to the in-fibre electrodes (see Fig. 2). $V_{1}$ and $V_{2}$ arethe voltages applied to the electrode pairswhich flank $\mathrm{LC}_{1}$ and $\mathrm{LC}_{2}$, respectively. The 
transmission axis of the linear polarizer which surrounds the fibrepointsin the z-axis. The width of the LC-microchannels is $30 \mu \mathrm{m}$, and their distance from the fibrecentreis 160 $\mu \mathrm{m}$ (see Fig. 2b), leading to an angular resolution of $\sim 11^{\circ}$ for this structure. (left and right side) In contrast to recording the far field laser intensity as in Fig. 4a, here we directly image the laser light at the plane of the LC-filled microchannels as the applied voltage $\mathrm{V}_{1}$ and $\mathrm{V}_{2}$ are varied. The light intensity passing through $\mathrm{LC}_{1}\left(\mathrm{LC}_{2}\right)$ does not change as a function of the voltage $V_{2}\left(V_{1}\right)$, which indicates the independent control available in each direction.(The randomly dispersed red dots seen in the microchannels with $V_{1}$ and $V_{2}$ at $0 \mathrm{~V}$ arise due toscattering of the laser light from a non-ideal alignment of the LCs.) b, Laser spectra measured for laser light transmission though $\mathrm{LC}_{2}$ as a function of $\mathrm{V}_{2}$.

Acknowledgments: A.M.S. is grateful to Prof. Federico Capasso, Prof. Ayman F. Abouraddy, Prof. Howard Stone, Prof. Zheng Wang, Dana Shemuly, Sylvain Danto, Daosheng Deng, Zachary Ruff, Nicholas Orf, and Alex Nemiroski for fruitful discussions. We appreciate Mark Stolyar for his help in engineering the first rapid prototype version of the fluidic /optical fibre coupler, and we thank Andrew Gallant at the MIT Central Machine Shop for producing the final version. Jelena Ryvkina is credited for the artwork in Supplementary Fig. S2. A.M.S. is thankful for the support from the US National Science Foundation Graduate Research Fellowship. L.W. acknowledges the support from Technical University of Denmark. This work was supported in part by the Materials Research Science andEngineering Program of the US National Science Foundation under award numberDMR-0819762 and also in part by the US Army Research Office throughthe Institute for SoldierNanotechnologies under contract number W911NF-07-D-0004. 
Author contributions:A.M.S., L.W., and O.S. plannedthe experiments. A.M.S. and L.W performed the experiments.A.M.S., L.W., and F.S.designed the fibre structures. A.M.S. and L.W. fabricated the fibres. O.S. and S.L.C. carried out simulations.A.M.S., L.W., O.S., F.S., Y.F. and J.D.J. conceived the presented ideas. A.M.S., J.D.J. and Y.F.co-wrote the manuscript. J.D.J. and Y.F. supervised the research. All authors analyzed the data.

Additional information: The authors declare no competing financial interests. 